\title{
Investigation of Mn-implanted n-type Ge
}

\author{
Lifeng Liu ${ }^{\mathrm{a}, *}$, Nuofu Chen ${ }^{\mathrm{a}, \mathrm{b}}$, Zhigang Yin ${ }^{\mathrm{a}}$, Fei Yang ${ }^{\mathrm{a}}$, \\ Jianping Zhou ${ }^{\mathrm{a}}$, Fuqiang Zhang ${ }^{\mathrm{a}}$ \\ ${ }^{a}$ Key Laboratory of Semiconductor Materials Science, Institute of Semiconductors, The Chinese Academy of Sciences, \\ Beijing 100083, China \\ ${ }^{\mathrm{b}}$ National Laboratory of Micro-Gravity, Institute of Mechanics, The Chinese Academy of Sciences, Beijing 100083, China
}

Received 16 January 2004; accepted 4 February 2004

Communicated by R. Kern

\begin{abstract}
$\mathrm{Mn}^{+}$irons were implanted to n-type Ge(1 111$)$ single crystal at room temperature with an energy of $100 \mathrm{keV}$ and a dose of $3 \times 10^{16} \mathrm{~cm}^{-2}$. Subsequently annealing was performed at $400^{\circ} \mathrm{C}$ for $1 \mathrm{~h}$ under flowing nitrogen gas. X-ray diffraction measurements show that as-implanted sample is amorphous and the structure of crystal is restored after annealing. Polycrystalline germanium is formed in annealed sample. There are no new phases found except germanium. The samples surface morphologies indicate that annealed sample has island-like feature while there is no such kind of characteristic in as-implanted sample. The elemental composition of annealed sample was analyzed by Auger electron spectroscopy. It shows that manganese ions are deeply implanted into germanium substrate and the highest manganese atomic concentration is $8 \%$ at the depth of $120 \mathrm{~nm}$. The magnetic properties of samples were investigated by an alternating gradient magnetometer. The annealed sample shows ferromagnetic behavior at room temperature.
\end{abstract}

(C) 2004 Elsevier B.V. All rights reserved.

PACS: $81.05 . \mathrm{Ea} ;$ 81.05.Zx; 81.15.Hi; 82.80.Pv

Keywords: A1. Auger electron spectroscopy; A1. X-ray diffraction; A3. Ion implantation; B2. Semiconducting germanium

\section{Introduction}

Diluted magnetic semiconductors (DMSs) combine the electronic transport properties of semiconductors and the memory characters of magnetic materials and are important to develop spin-controlled electronic devices and integrated magnetic device applications. Nowadays, the

\footnotetext{
*Corresponding author. Tel.: + 86-10-8230-4569; fax: + 8610-8230-4469.

E-mail address: 1fliu@red.semi.ac.cn (L. Liu).
}

investigation of DMSs has received a great deal of attention. The most extensively studied and most thoroughly understood materials of DMSs are $\mathrm{A}_{1-x}^{\mathrm{II}} \mathrm{Mn}_{x} \mathrm{~B}^{\mathrm{VI}}$ and $\mathrm{A}_{1-x}^{\mathrm{III}} \mathrm{Mn}_{x} \mathrm{~B}^{\mathrm{V}}$, in which $\mathrm{Mn}$ replaces a fraction of groups II or III sublattices randomly [1-4]. But, group IV (such as Si and Ge)based DMSs may result more practical for applications since present semiconductor technology may be used [5-7].

Some reports about Ge-based DMSs were presented in the last years. Park et al. [7] reported on the epitaxial growth of $\mathrm{Mn}_{x} \mathrm{Ge}_{1-x}$ by molecular 
beam epitaxy (MBE), in which the Curie temperature is found to increase linearly with manganese $(\mathrm{Mn})$ concentration from 25 to $116 \mathrm{~K}$. Sunglae Cho et al. [8] reported the ferromagnetism in highly Mn-doped (up to 6\%) Ge single crystals with $T_{\mathrm{c}}=285 \mathrm{~K}$. Franco D'Orazio et al. [9] reported the magnetic properties of Mn-implanted $\mathrm{Ge}$ in which a hysteresis appearing just below room temperature was experimentally observed. A hybrid structure Ge:Mn was reported showing ferromagnetic properties and magnetoresistance phenomena near room temperature, attributed to $\mathrm{Mn}$-rich ferromagnetic clusters [10]. However, Gebased DMSs have not been fully investigated yet.

In this paper, the characteristics of Mn-implanted n-type Ge were investigated. The structure and surface morphology of samples were analyzed by X-ray diffraction (XRD) and atomic force microscopy (AFM) while the atomic concentration of sample dependence on the depth was studied by Auger electron spectroscopy (AES). The magnetic properties of samples were investigated by an alternating gradient magnetometer (AGM) at room temperature.

\section{Material preparation}

Samples investigated in this paper were obtained by implantation $\mathrm{Mn}^{+}$ions into (1 111 )-oriented $\mathrm{n}$ type Ge single crystal substrate and subsequently annealing. The $\mathrm{Ge}$ substrate was chemically cleaned using a standard $\mathrm{HF} / \mathrm{H}_{2} \mathrm{O}$ etchant and $\mathrm{H}_{2} \mathrm{O}_{2} / \mathrm{H}_{2} \mathrm{O}$ rinse before sample growth. Then, the Ge substrate was delivered into growth chamber where the vacuum was $<1 \times 10^{-5} \mathrm{~Pa}$. $\mathrm{Mn}^{+}$irons were uniformly implanted to substrate with an energy of $100 \mathrm{keV}$ and a dose of $3 \times 10^{16} \mathrm{~cm}^{-2}$ while the temperature of the substrate during the process was kept at room temperature (sample A). Following implantation, part of the as-implanted sample was annealed at $400^{\circ} \mathrm{C}$ for $1 \mathrm{~h}$ under a flowing nitrogen gas (sample B). During the annealing process the sample surface was attached to a polished germanium wafer so as to prevent manganese from evaporating from the sample surface.

\section{Measurements and analyses}

\subsection{Structural analyses}

The structural properties of samples were studied by XRD measurements. The XRD patterns of samples were measured with a Rigaku diffractometer using $\mathrm{Cu} \mathrm{K} \alpha$ radiation for structural analyses. The wide range XRD $\left(20-80^{\circ}\right)$ patterns are shown in Fig. 1. The XRD spectra of samples A and B are Figs. 3(a) and (b), respectively. In sample $A$, only $\operatorname{Ge}\left(\begin{array}{lll}1 & 1 & 1\end{array}\right)$ peak is observed. The diffraction peaks of $\mathrm{Ge}(220)$ and (311) appear in sample B. The results show that the crystal structure of the as-implanted sample is destroyed by the heavily manganese ions


Fig. 1. XRD spectra of samples: (a) sample A, (b) sample B and (c) $\mathrm{Ge}\left(\begin{array}{lll}1 & 1 & 1\end{array}\right)$ X-ray rocking curve for sample B. 
implanting and is amorphous. After annealing at $400^{\circ} \mathrm{C}$, the crystal structure of the as-implanted sample is restored and polycrystalline germanium is formed due to the incorporation of a lot of manganese ions. No additional diffraction peaks can be found except germanium both in asimplanted sample and in annealed sample. We have carefully searched for secondary phases such as $\mathrm{MnGe}$ compounds. However, none were observable. Then we measured samples by the Xray double-crystal diffractometer. The X-ray diffractometer used is Philips X'pert-MRD (X'Pert Materials Research Diffractometer System) equipped with a multipurpose sample stage. The DXRD profiles were measured by $\omega-2 \theta$ scanning, in which again there are only germanium peaks. The DXRD patterns are not shown here. These XRD dates show there are no new phases formed in samples. To study more carefully, sample B was scanned slowly near $\mathrm{Ge}\left(\begin{array}{lll}1 & 1 & 1\end{array}\right)$ reflection. Fig. 1(c) shows the rocking curve around $\mathrm{Ge}\left(\begin{array}{lll}1 & 1 & 1\end{array}\right)$. It can be seen that the spectrum of $\mathrm{Ge}(111)$ is asymmetric and extended to lowangular side of the peak, which indicates that the lattice of germanium is expanded because germanium is implanted by the larger manganese atoms and shows the inhomogeneous concentration of manganese in depth.

\subsection{Morphological analyses}

AFM is a noninvasive technique to deliver three-dimensional realistic impressions of the measured sample surface. It is an easy and fast method to show the change in the amplitude of the surface roughness. The surface morphologies of samples were observed by AFM. Figs. 2(a) and (b) show the surface morphology of samples A and B, respectively. From Fig. 2, it can be seen that the surface morphology of samples A and B is quite different. The AFM image of sample B shows island-like feature that uniformly cover the sample surface while there is no such kind of characteristic at the surface of sample A. The AFM results indicate that the crystal structure of sample $\mathrm{A}$ is amorphous while the crystal structure of sample B is crystallized. The AFM results are consistent with the XRD profiles.
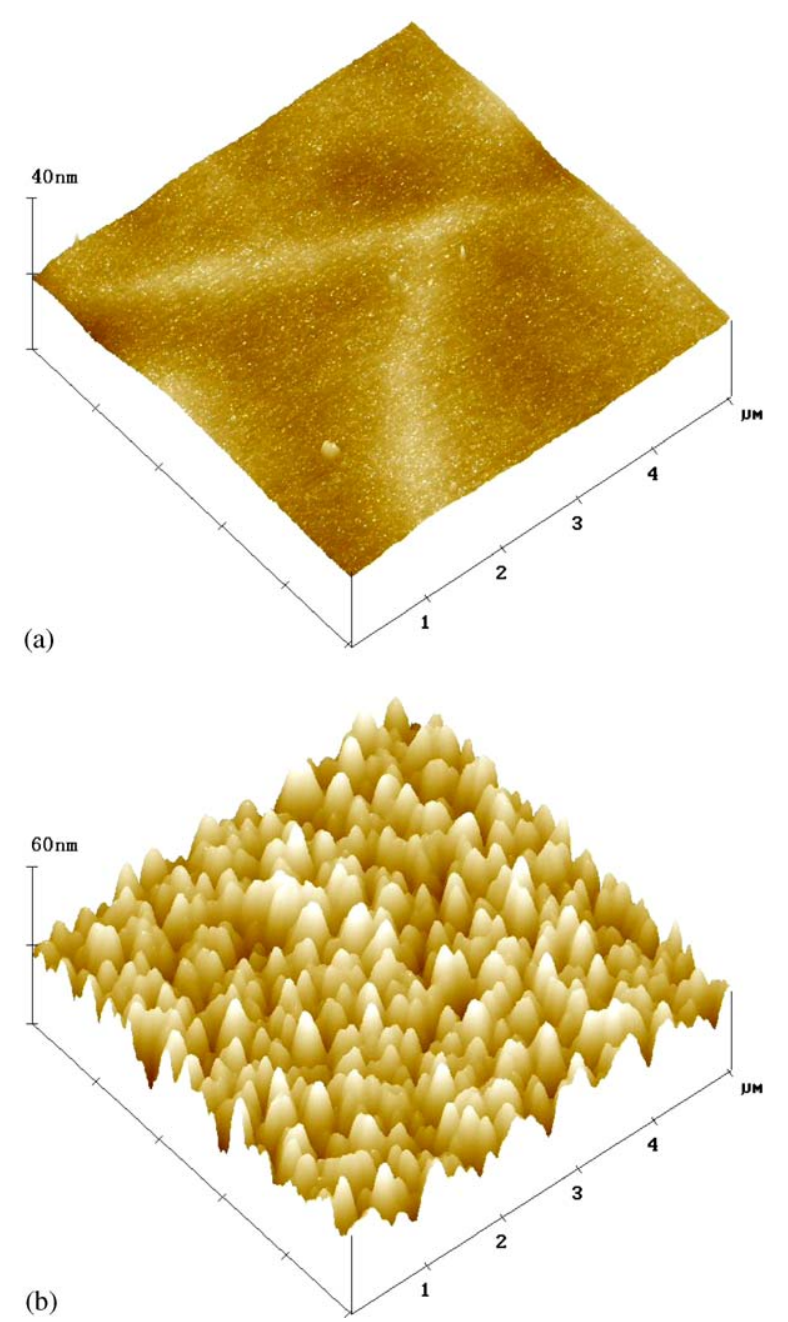

Fig. 2. AFM morphologies of samples: (a) sample A and (b) sample B.

\subsection{Compositional analyses}

The PHI-610/SAM AES system was employed for analyzing the compositional variation of sample. Fig. 3(a) is the AES spectra at the surface of sample B, which show that there are manganese, germanium, carbon and oxygen at the surface of sample B. Sample B depth profile is shown in Fig. 3(b). It can be seen that the concentrations of the oxygen and carbon are very high at the sample surface. The concentration of the carbon decreases rapidly along the depth. The reason for existing of carbon is that sample B surface was contaminated 

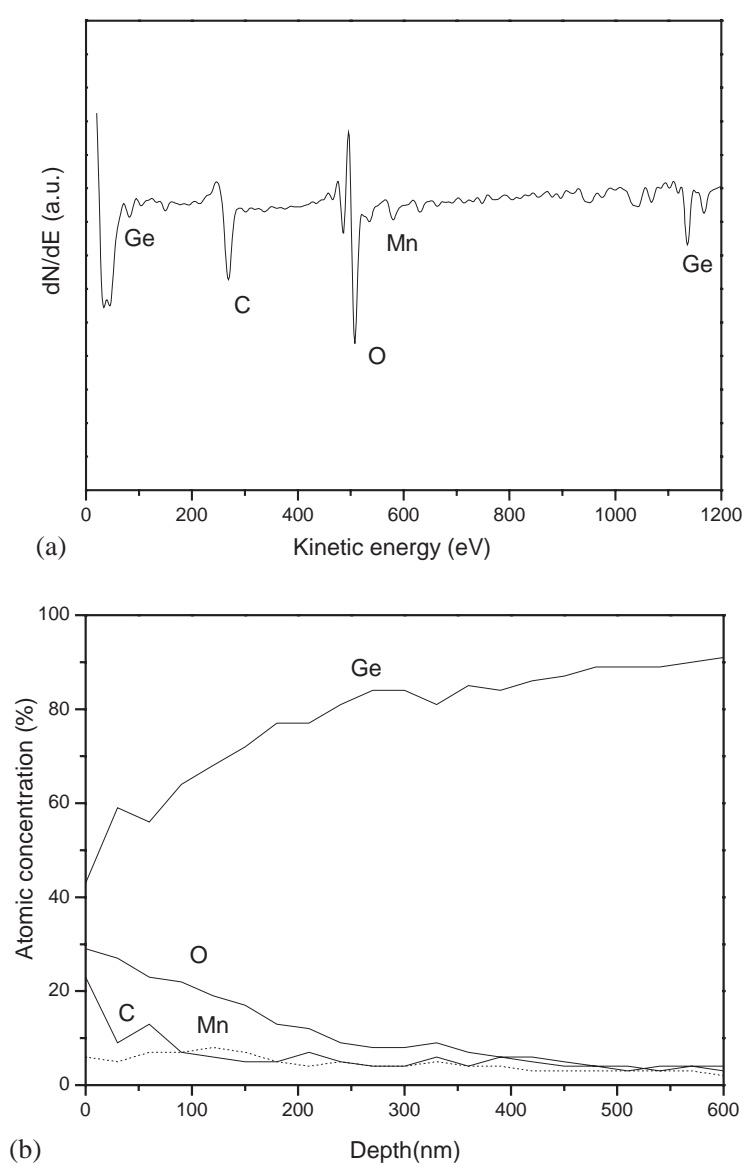

Fig. 3. Auger electron spectroscopy of sample B: (a) at the sample surface and (b) sample depth profile.

after it was taken out from the growth chamber. The concentration of the oxygen decreases slowly along the depth. It is because that the oxygen atoms at the surface of the substrate diffuse with manganese ions while implanting. Manganese ions were deeply implanted into germanium substrate. The highest manganese atomic concentration of sample B is $8 \%$ at the depth of $120 \mathrm{~nm}$. After the depth of $120 \mathrm{~nm}$, the concentration of the manganese decreases along the depth. During the analyzed depth area, the relative concentration of germanium and manganese in sample B does not monotonically increase or decrease, indicating that there is a segregation of manganese atoms in the annealed sample.

\subsection{Magnetic analyses}

The magnetic properties of the samples were measured on a Model 2900 MicroMag $^{\text {TM }}$ AGM at room temperature. The results of the magnetization measurements by AGM reveal room-temperature ferromagnetism for sample $\mathrm{B}$ while no ferromagnetism can be observed for sample A. Fig. 4 shows the ferromagnetic hysteresis loop of sample B. The magnetization curve is obtained with the applied field parallel to the plane of sample. The diamagnetic background contribution due to $\mathrm{Ge}$ is subtracted from the data. The coercivity $H_{\mathrm{C}}$ and saturation magnetization $M_{\mathrm{S}}$ of sample B are about $408 \mathrm{Oe}$, and $5.62 \times 10^{-5} \mathrm{emu}$, respectively.

Pure Ge does not have a net magnetic moment, resulting in diamagnetism with a molar susceptibility of $-11.6 \times 10^{-6} \mathrm{~cm}^{3} \mathrm{~mol}^{-1}$ [11]. Metallic $\mathrm{Mn}$ is antiferromagnetic, with a Néel temperature of $100 \mathrm{~K}$ [12]. In addition, nearly all of the possible $\mathrm{Mn}$-based binary and ternary oxide candidates are antiferromagnetic. Only the $\mathrm{Mn}_{3} \mathrm{O}_{4}$ is ferromagnetic with a Curie temperature of $42 \mathrm{~K}$ [12]. However, $\mathrm{Mn}_{3} \mathrm{O}_{4}$ could not account for the room-temperature ferromagnetic behavior of sample $\mathrm{B}$ due to its low Curie temperature. DMS $\mathrm{Mn}_{x} \mathrm{Ge}_{1-x}$ is ferromagnetic and has a large variation of Curie temperature from 25 to $285 \mathrm{~K}$ according to different reports $[7,8]$. The estimated Curie temperature of $\mathrm{Mn}_{x} \mathrm{Ge}_{1-x}$ is up to $400 \mathrm{~K}$ [13]. From the XRD results, no new phases are

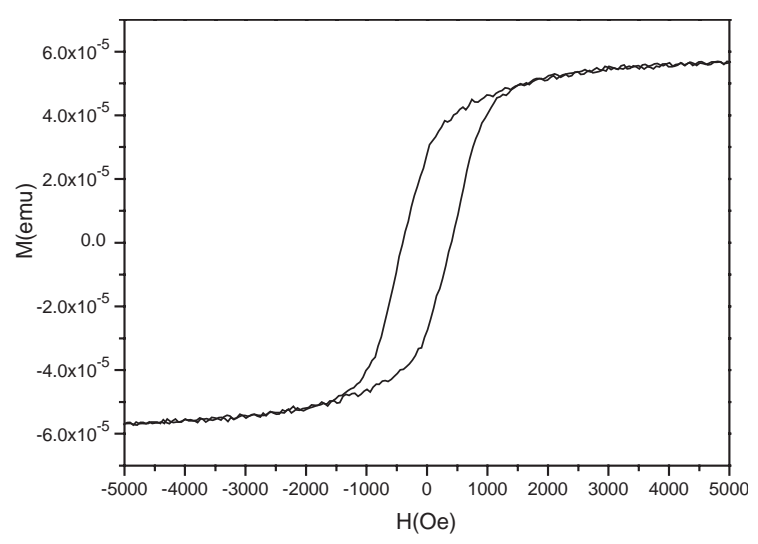

Fig. 4. Ferromagnetic hysteresis loop of sample B measured by AGM at room temperature. 
found. It is possible that $\mathrm{Mn}_{x} \mathrm{Ge}_{1-x}$ structure is formed after the annealing process and is responsible for the ferromagnetism of sample B. Furthermore, the dose of manganese ions in this study is above the solid solubility limit of $\mathrm{Mn}$ in Ge. It can be seen from AES depth profile of sample B that there is a segregation of manganese atoms. So, it is also possible that Mn-related ferromagnetic clusters such as $\mathrm{Mn}_{5} \mathrm{Ge}_{3}$ and $\mathrm{Mn}_{11} \mathrm{Ge}_{8}$ with Curie temperatures near room temperature $[14,15]$ are formed in annealed sample. We cannot make sure what phase is responsible for the ferromagnetism of the annealed sample before making further investigation on it.

\section{Summary}

The characteristics of Mn-implanted n-type germanium were investigated. The structure of as-implanted sample is amorphous. As-implanted sample was annealed at $400^{\circ} \mathrm{C}$ and crystallized. Polycrystalline germanium is formed. There are no new phases found except germanium both in asimplanted sample and in annealed sample. Annealed sample AES depth profile shows that manganese ions are deeply implanted into germanium substrate and the highest manganese atomic concentration is $8 \%$ at the depth of $120 \mathrm{~nm}$. Room-temperature ferromagnetic behavior shows in the annealed sample though further investigation is still needed.

\section{Acknowledgements}

This work was partially supported by the National Natural Science Foundation of China
(Grant Nos. 60176001, 60390072) and Special Funds for Major State Basic Research Projects of China (Grant Nos. 20000365, 2002CB311905).

\section{References}

[1] H. ohno, A. Shen, F. Matsukura, A. Oiwa, A. Endo, S. Katsumoto, Y. Iye, Appl. Phys. Lett. 69 (1996) 363.

[2] M.L. Reed, N.A. El-Masry, H.H. Stadelmaier, M.K. Ritums, M.J. Reed, C.A. Parker, J.C. Roberts, S.M. Bedair, Appl. Phys. Lett. 79 (2001) 3473.

[3] A. Haury, A. Wasiela, A. Arnoult, J. Cibert, S. Tatarenko, T. Dietl, Y. Merle, D. Aubigné, Phys. Rev. Lett. 79 (1997) 511.

[4] X. Liu, U. Bindley, Y. Sasaki, J.K. Furdyna, J. Appl. Phys. 91 (2002) 2859.

[5] Hiroshi Nakayamaa, Hitoshi Ohtab, Erkin Kulatovc, Physica B 302-303 (2001) 419.

[6] A. Stroppa, S. Picozzi, A. Continenza, A.J. Freeman, Phys. Rev. B 68 (2003) 155203.

[7] Y.D. Park, A.T. Hanbicki, S.C. Erwin, C.S. Hellberg, J.M. Sullivan, J.E. Mattson, T.F. Ambrose, A. Wilson, G. Spanos, B.T. Jonker, Science 295 (2002) 651.

[8] Sunglae Cho, Sungyoul Choi, Soon Cheol Hong, Yunki Kim, Phys. Rev. B 66 (2002) 033303.

[9] Franco D’Orazio, Franco Lucari, Maurizio. Passacantando, Pietro Picozzi, Sandro Santucci, Adriano. Verna, IEEE Trans. Magn. 38 (2002) 2856.

[10] Y.D. Park, A. Wilson, A.T. Hanbicki, J.E. Mattson, T. Ambrose, G. Spanos, B.T. Jonker, Appl. Phys. Lett. 78 (2001) 2739.

[11] Sungyoul. Choi, Soon Cheol Hong, Sunglae Cho, Yunki Kim, Bong-Jun Kim, Y.C. Kim, Appl. Phys. Lett. 81 (2002) 3606.

[12] D.P. Norton, S.J. Pearton, A.F. Hebard, N. Theodoropoulou, L.A. Boatner, R.G. Wilson, Appl. Phys. Lett. 82 (2003) 239.

[13] Yu-Jun. Zhao, Tatsuya Shishidou, A.J. Freeman, Phys. Rev. Lett. 90 (2003) 047204.

[14] K. Kanematsu, J. Phys. Soc. Jpn. 17 (1962) 85.

[15] N. Yamada, K. Maeda, Y. Usami, T. Ohoyama, J. Phys. Soc. Jpn. 55 (1986) 3721. 